05

\title{
Диэлектрическая спектроскопия как метод исследования тонких пленок диоксида ванадия
}

\author{
(C) А.В. Ильинский, ${ }^{1}$ Р.А. Кастро, ${ }^{2}$ М.Э. Пашкевич, ${ }^{3}$ Е.Б. Шадрин ${ }^{1}$ \\ ${ }^{1}$ Физико-технический институт им. А.Ф. Иофффе РАН, \\ 194021 Санкт Петербург, Россия \\ ${ }^{2}$ Российский государственный педагогический университет им. А.И. Герцена, \\ 191186 Санкт-Петербург, Россия \\ ${ }^{3}$ Санкт-Петербургский политехнический университет Петра Великого, \\ 195251 Санкт-Петербург, Россия \\ e-mail: shadr.solid@mail.ioffe.ru
}

Поступило в Редакцию 6 мая 2019 г.

В окончательной редакции 6 мая 2019 г.

Принято к публикации 27 мая 2019 г.

Исследованы диэлектрические спектры образцов тонких (1200 А) пленок диоксида ванадия — материала с сильными электрон-электронными корреляциями. Исследованы как нелегированные, так и легированные германием пленки $\mathrm{VO}_{2}: \mathrm{Ge}$. Для последних обнаружен дополнительный максимум в частотном спектре тангенса угла диэлектрических потерь. Изучена тонкая структура спектров и выполнена физическая интерпретация двух максимумов на частотной зависимости тангенса угла диэлектрических потерь и двух полуокружностей на Коул-Коул диаграмме. Анализ результатов произведен на основе эквивалентных электрических схем образцов: одноконтурной RC-схемы для случая нелегированной пленки $\mathrm{VO}_{2}$ и двухконтурной — для $\mathrm{VO}_{2}: \mathrm{Ge}$. Определены численные значения параметров модельных схем.

Ключевые слова: диэлектрическая спектроскопия, диоксид ванадия $\mathrm{VO}_{2}$, легированные пленки $\mathrm{VO}_{2}: \mathrm{Ge}$, корреляционные эффекты, тангенс угла диэлектрических потерь.

DOI: $10.21883 /$ JTF.2019.12.48487.189-19

\section{Введение}

В настоящее время сохраняется интерес исследователей к информации, даваемой методом диэлектрической спектроскопии [1 3]. Это связано как с относительной простотой методики, так и с наличием компьютеризованных диэлектрических спектрометров высокого качества, оснащенных пакетом высокоскоростных программ обработки результатов измерений. Важно, что методы диэлектрической спектроскопии применимы для анализа свойств объектов различной природы: твердых и жидких тел, органических и неорганических материалов. И, в частности, метод применим к тонким пленкам, синтезированным на твердых непроводящих подложках.

Настоящая работа посвящена моделированию электрических процессов, протекающих в тонких пленках диоксида ванадия - материала с сильными электронэлектронными корреляциями [4] и является продолжением предыдущей работы авторов [5]. Исследовались как нелегированные, так и легированные германием пленки $\mathrm{VO}_{2}$. Оказалось, что моделирование электрического отклика нелегированных пленок $\mathrm{VO}_{2}$ не представляет особых трудностей, тогда как моделирование отклика легированных пленок требует привлечения для своего анализа многоконтурных эквивалентных схем и более трудоемких, чем в предыдущем случае, расчетов на их основе.

\section{1. Методика эксперимента}

В настоящей работе использовался спектрометр марки „Alpha-Beta Impedance Analyzer“ фирмы Novocontrol Technologies, позволявший проводить измерения частотных зависимостей модуля амплитуды $I_{0}(f)$ комплексного тока $I^{*}(f)$, протекающего через образец, и разности фаз $\phi(f)$ между током и приложенным опорным напряжением $U$. Диапазон изменения частоты $f$ составлял $10^{1}-10^{6} \mathrm{~Hz}$. С помощью встроенного в спектрометр конвертера эти зависимости преобразовывались в частотные зависимости комплексного импеданса $Z^{*}(f)$, комплексной диэлектрической проницаемости $\varepsilon^{*}(f)$ и тангенса угла диэлектрических потерь $\operatorname{tg} \delta(f)$, где $\delta=\pi / 2-\phi$.

Измерительная ячейка спектрометра, в которую помещался исследуемой образец, представляла собой изолирующий цилиндр с двумя плоскими металлическими электродами площадью $S=0.79 \mathrm{~cm}^{2}$ каждый. Расстояние $d$ между электродами определялось толщиной изолирующей подложки, на которую была нанесены пленка, и составляло $d=0.50 \mathrm{~mm}$ для подложек с нелегированными пленками $\mathrm{VO}_{2}$ и $d=0.60 \mathrm{~mm}$ для подложек с легированными пленками $\mathrm{VO}_{2}: \mathrm{Ge}$. Геометрическая (без образца) емкость ячейки $C_{0}=\varepsilon_{0} \cdot S / d$ в наших экспериментах составляла $1.40 \mathrm{pF}$ и $1.17 \mathrm{pF}$ соответственно.

Нелегированные пленки $\mathrm{VO}_{2}$ синтезировались на ситалловой подложке методом катодного распыления V- 
мишени с последующим окислением паров металла в потоке кислорода вблизи подложки. Легированные германием пленки $\mathrm{VO}_{2}$ : Ge синтезировались на ситалловой подложке методом взрывной сублимации стехиометричной смеси порошков $\mathrm{VO}_{2}$ и $\mathrm{GeO}_{2}$. Площадь поверхности пленочных образцов составляла $\sim 1 \mathrm{~cm}^{2}$, толщина нелегированных пленок равнялась $1100 \AA$, толщина легированных - $1400 \AA$.

При анализе диэлектрических спектров мы исходили из того, что электрический отклик образца при подаче на измерительную ячейку синусоидального напряжения частоты $\omega$ и амплитуды $U_{o}$ является линейным. Соотношение между амплитудой напряжения $U_{o}$ и амплитудой $I_{o}$ протекающего через образец тока, а также разность фаз $\phi$ колебаний между током и напряжением, определяются физическими свойствами объекта, которые и являются предметом исследований.

Линейность отклика позволяет применить символический метод расчета путем представления частотных зависимостей тока и напряжения в комплексной форме [6]:

$$
\begin{gathered}
U(t)=U_{0} \sin (\omega t)=\operatorname{Im}\left[U^{*} \exp (i \omega t)\right], \\
I(t)=I_{o} \sin (\omega t+\phi)=\operatorname{Im}\left[I^{*} \exp (i \omega t)\right],
\end{gathered}
$$

где символы $U^{*}$ и $I^{*}$ представляют собой комплексные амплитуды напряжения и тока соответственно. Мы полагаем $U^{*}=U_{o}$ и, выделяя в явном виде вещественные и мнимые части комплексных величин, можем записать $I^{*}=I_{o} \exp (i \phi)=I_{o} \cos (\phi)+i I_{o} s \sin (\phi)$, где $I_{o}=\left(I^{\prime}+I^{\prime \prime}\right)^{1 / 2}$, а $i-$ мнимая единица. Исходя из комплексной формы закона Ома $I^{*}(t)=U^{*}(t) / Z^{*}$, получаем $Z^{*}=\left[U_{o} \exp (i \omega t)\right] /\left[I^{*} \exp (i \omega t)\right]$, т.е. $\left(Z^{\prime}+i Z^{\prime \prime}\right)=$ $=U_{o} /\left(I^{\prime}+i I^{\prime \prime}\right)$ или $Z^{\prime}=\left(U_{o} / I_{o}\right) I^{\prime}$ и $Z^{\prime}=-\left(U_{o} / I_{o}\right) I^{\prime \prime}$.

Конструкция диэлектрического спектрометра предусматривает измерение вещественных амплитуд $U_{o}$ и $I_{o}$, а также тангенса угла между вектором полного импеданса образца и вещественной осью, причем этот угол совпадает с углом $\phi$ разности фаз между колебаниями тока через образец и колебаниями подаваемого на образец напряжения. Поэтому $\operatorname{tg} \phi=I^{\prime \prime} / I^{\prime}$ или с учетом выражений $Z^{\prime}=\left(U_{o} / I_{o}\right) I^{\prime}$ и $Z^{\prime \prime}=-\left(U_{o} / I_{o}\right)^{\prime \prime} \operatorname{tg} \phi=-Z^{\prime \prime} / Z^{\prime}$.

Как указано выше, $I_{o}=\left(I^{\prime}\right)^{2}+\left(I^{\prime \prime}\right)^{2}$ и $\operatorname{tg} \phi=I^{\prime \prime} / I^{\prime}$, откуда получаем $I^{\prime}=I_{o} /\left[1+(\operatorname{tg} \phi)^{2}\right]^{-1 / 2}$ и $I^{\prime \prime}=\left(I_{o} \operatorname{tg} \phi\right) /$ $/\left[1+(\operatorname{tg} \phi)^{2}\right]^{-1 / 2}$, где $I_{o}$ и $\phi-$ экспериментальные величины, измеряемые спектрометром для каждой частоты $\omega=2 \pi f$ колебаний входного напряжения, причем $f$ изменяется в диапазоне $f=10^{1}-10^{6} \mathrm{~Hz}$. Это позволяет экспериментально определить вещественную и мнимую части комплексного импеданса $Z^{*}$ согласно выражениям $Z^{\prime}=\left(U_{o} / I_{o}\right) I^{\prime}$ и $Z^{\prime \prime}=-\left(U_{o} / I_{o}\right) I^{\prime \prime}$.

Конструкция измерительной ячейки спектрометра такова, что индуктивная составляющая полного импеданса пленочного образца пренебрежимо мала, откуда следует, что при анализе частотной зависимости реактивной составляющей комплексного импеданса $Z^{*}$ может учитываться только комплексная электрическая емкость $C^{*}$ измерительной ячейки, содержащей образец.
Поэтому в рассмотрение вводится комплексная диэлектрическая проницаемость образца $\varepsilon^{*}=\varepsilon^{\prime}+i \varepsilon^{\prime \prime}$, получаемая из соотношения $Z^{*}=1 /\left(i \omega C^{*}\right)=1 /\left(i \omega C_{o} \varepsilon^{*}\right)$, после чего простые выкладки приводят к выражению $\varepsilon^{\prime}+i \varepsilon^{\prime \prime}=1 /\left(-\omega C_{o} Z^{\prime}+i \omega C_{o} Z^{\prime}\right)$. Избавляясь от мнимости в знаменателе и приравнивая вещественные и мнимые части $\varepsilon^{*}$, получаем соотношения

$$
\begin{gathered}
\varepsilon^{\prime}=-Z^{\prime \prime} /\left\{\omega C_{0}\left[\left(Z^{\prime \prime}\right)^{2}+\left(Z^{\prime}\right)^{2}\right]\right\}, \\
\varepsilon^{\prime \prime}=Z^{\prime} /\left\{\omega C_{o}\left[\left(Z^{\prime \prime}\right)^{2}+\left(Z^{\prime}\right)^{2}\right]\right\},
\end{gathered}
$$

где $Z^{\prime}$ и $Z^{\prime \prime}$ - вещественная и мнимая части комплексного импеданса образца, определяемые, таким образом, для каждой частоты $\omega$ колебаний входного напряжения.

C учетом выражений $Z^{\prime}=\left(U_{o} / I_{o}\right) I^{\prime}$ и $Z^{\prime \prime}=-\left(U_{o} / I_{o}\right) I^{\prime \prime}, \quad$ а также выражений $\quad I^{\prime}=I+{ }_{o} /\left[1+(\operatorname{tg} \phi)^{2}\right]^{-1 / 2}$ $I^{\prime \prime}=\left(I_{o} \operatorname{tg} \phi\right) /\left[1+(\operatorname{tg} \phi)^{2}\right]^{-1 / 2}$, получим связь между $\varepsilon^{\prime}, \varepsilon^{\prime \prime}$ и измеряемыми величинами $I_{o}, U_{o}$ и $\phi$ :

$$
\begin{gathered}
\left.\left|\mathcal{\varepsilon}^{\prime}\right|=\left(I_{o} \operatorname{tg} \phi\right) /\left[\omega C_{o} U_{o}\right)\left(1+(\operatorname{tg} \phi)^{2}\right)^{-1 / 2}\right], \\
\left.\left|\varepsilon^{\prime \prime}\right|=\left(I_{o}\right) /\left[\omega C_{o} U_{o}\right)\left(1+(\operatorname{tg} \phi)^{2}\right)^{-1 / 2}\right],
\end{gathered}
$$

Таким образом, метод диэлектрической спектроскопии позволяет экспериментально определить частотные зависимости (диэлектрические спектры) вещественной $\varepsilon^{\prime}(\omega)$ и мнимой $\varepsilon^{\prime \prime}(\omega)$ частей комплексной диэлектрической проницаемости материала вне зависимости от конкретной эквивалентной электрической схемы образца (при отсутствии индуктивной составляющей).

С другой стороны, приведенные выражения (1), (2) позволяют выполнить процедуру моделирования электрического отклика образца путем использования его эквивалентной электрической схемы, в рамках которой могут быть найдены аналитические выражения, связывающие величины $Z^{\prime}$ и $Z^{\prime \prime}$ с конкретными физическими параметрами пленки диоксида ванадия. Вслед за этим путем сравнения экспериментальных спектров со спектрами, вычисленными в рамках модельной эквивалентной схемы, может быть проверена правомочность применения той или иной схемы, а также определены численные значения параметров модельной эквивалентной схемы.

\section{2. Результаты исследования диэлектрических спектров пленок $\mathrm{VO}_{2}$ и $\mathrm{VO}_{2}: \mathrm{Ge}$}

На рис. 1, 2 наборами точек представлены полученные при комнатной температуре экспериментальные частотные зависимости тангенса угла диэлектрических потерь $\operatorname{tg} \delta(f)$ и Коул-Коул диаграммы $\varepsilon^{\prime \prime}\left(\varepsilon^{\prime}\right)$ для тонких пленок диоксида ванадия. Здесь угол $\delta=\pi / 2-\phi$. Сплошные линии - расчетные зависимости (см. ниже разд. 4).

Эксперименты показывают, что в случае нелегированных пленок $\mathrm{VO}_{2}$ наблюдается один максимум функции 

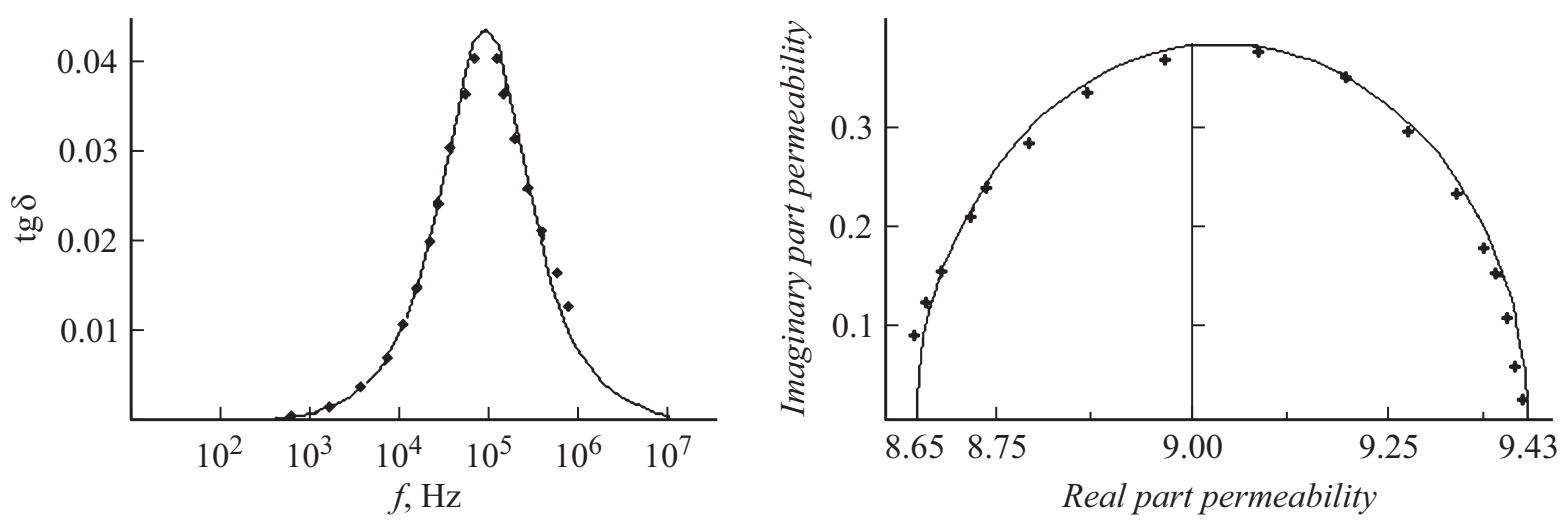

Рис. 1. $\operatorname{tg} \delta(f)$ и Коул-Коул диаграммы для нелегированных пленок $\mathrm{VO}_{2}$. Результаты измерений - точки, результаты расчета по схеме 3 - сплошные кривые. В расчете по схеме $3: C_{a}=170 \mathrm{pF}, C_{b}=13 \mathrm{pF}, R=10 \mathrm{k} \Omega$, геометрическая емкость ячейки $C_{o}=1.40 \mathrm{pF}$.
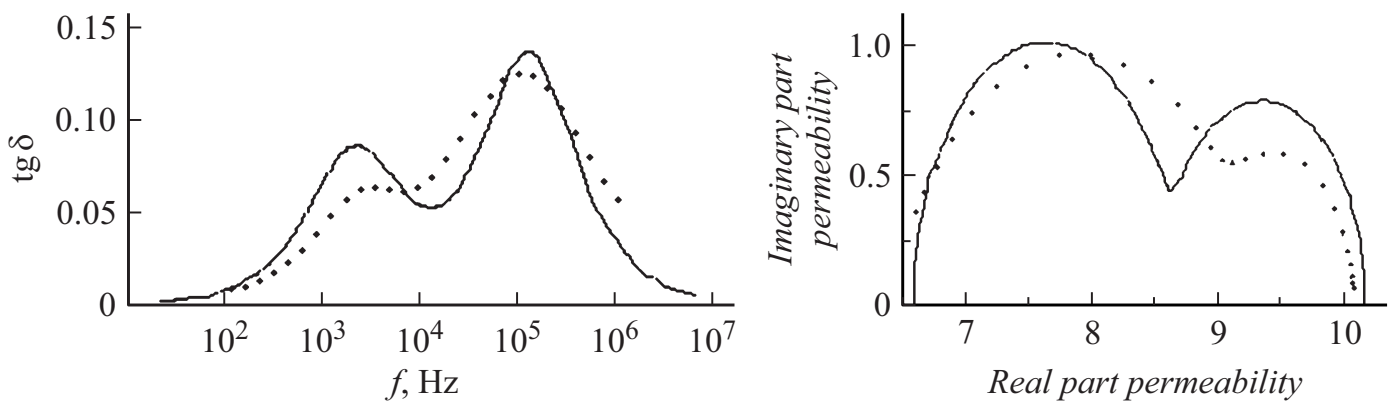

Рис. 2. $\operatorname{tg} \delta(f)$ и Коул-Коул диаграммы для легированных пленок $\mathrm{VO}_{2}$ : Ge. Результаты измерений - точки, результаты расчета по схеме 4 - сплошные кривые. В расчете по схеме 4: $C_{a}=15 \mathrm{pF}, C_{b}=7 \mathrm{pF}, R=60 \mathrm{k} \Omega, C_{m}=5 \mathrm{pF}, C_{n}=12 \mathrm{pF}, r=3 \mathrm{M} \Omega$, геометрическая емкость ячейки $C_{o}=1.17 \mathrm{pF}$.

$\operatorname{tg} \delta(f)$ на частоте $f_{0}=10^{5} \mathrm{~Hz}$, а также одна полуокружность на Коул-Коул диаграмме (рис. 1). Для легированных пленок $\mathrm{VO}_{2}$ : Ge спектры выглядят сложнее: на частотной зависимости $\operatorname{tg} \delta(f)$ наблюдаются два максимума при частотах $f_{01}=10^{5} \mathrm{~Hz}$ и $f_{02}=4 \cdot 10^{3} \mathrm{~Hz}$ и комплексная Коул-Коул диаграмма с двумя полуокружностями (рис. 2).

\section{3. Моделирование электрического отклика пленок $\mathrm{VO}_{2}$ : эквивалентные схемы, обоснование выбора}

Мы полагаем, что полученные экспериментально диэлектрические спектры следует анализировать на основе эквивалентной схемы, выбранной на базе следующих физических соображений.

А именно для исследованных в настоящей работе образцов следует принять во внимание электроемкость подложки и электроемкость тонкой пленки $\mathrm{VO}_{2}$, а также электрическое сопротивление пленки. Как указано выше, учитывать индуктивность рабочей ячейки спектрометра с исследуемым образцом и электрическое сопротивление подложки нет необходимости.
В разд. 3 мы рассмотрим четыре эквивалентные схемы (рис. 3): простую последовательную $R C$-цепочку -1 , простую параллельную $R C$-цепочку -2 , одноконтурную $R C$-схему -3 и двухконтурную $R C$-схему -4 .

Для схем $1-3 C_{b}$ представляет собой емкость подложки, $C_{a}$ и $R$ представляет собой емкость и сопротивление нелегированной пленки $\mathrm{VO}_{2}$.

Для случая легированной $\mathrm{Ge}$ пленки $\mathrm{VO}_{2}$ результаты эксперимента (два максимума $\operatorname{tg} \delta$ и две полуокружности на Коул-Коул диаграмме) вынуждают принять в качестве модельной двухконтурную схему 4. Здесь элементы схемы $C_{a}, C_{b}$ и $R$ соответствуют высокочастотному максимуму функции $\operatorname{tg} \delta \omega$ и высокочастотной полуокружности Коул-Коул диаграммы. Элементы схемы $C_{m}, C_{n}$ и $r$ соответствуют низкочастотному максимуму функции $\operatorname{tg} \delta(\omega)$ и низкочастотной полуокружности Коул-Коул диаграммы (рис. 2).

\section{4. Математическое моделирование для каждой схемы}

\section{1. Простая последовательная цепочка}

Для простой последовательной цепочки (рис. $3-1)$ имеем $Z^{*}=R+1 /\left(i \omega C_{b}\right), \operatorname{tg} \delta=1 /\left(\omega R C_{b}\right)$, а $\varepsilon^{\prime}$ и $\varepsilon^{\prime \prime}$ 
1

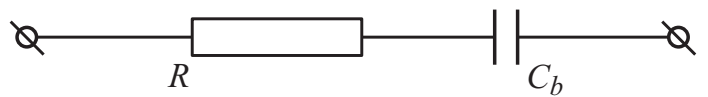

2

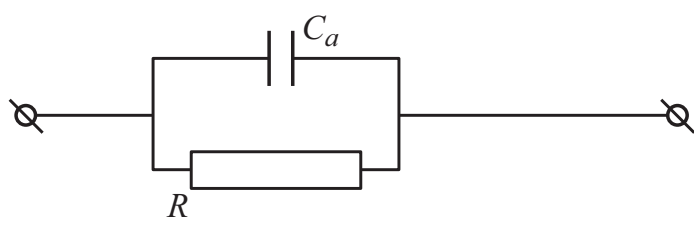

3

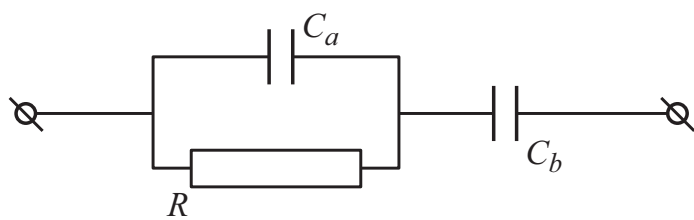

4

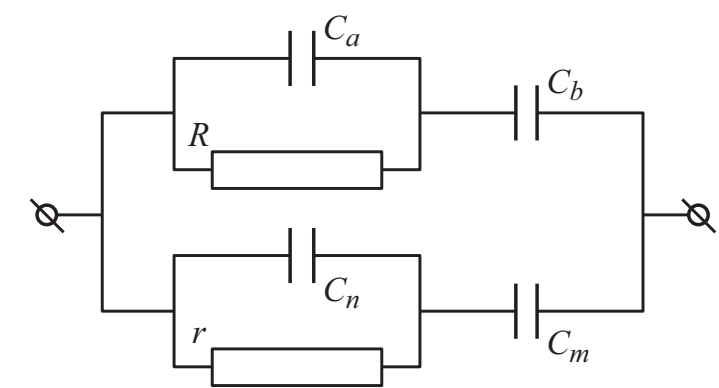

Рис. 3. Эквивалентные схемы для анализа данных диэлектрической спектроскопии.

даются выражениями (1), (2), где $\omega=2 \pi f$. Из этих выражений следует, что такая схема не соответствует результатам эксперимента, так как частотная зависимость $\operatorname{tg} \delta(f)$ не имеет максимума.

\section{2. Простая параллельная цепочка}

Для простой параллельной цепочки (рис. 3 - 2) имеем

$$
\begin{aligned}
& Z^{*}=Z^{\prime}+i Z^{\prime \prime}=R /\left(1+R^{2} \omega^{2} C_{a}^{2}\right) \\
& +i\left(R^{2} \omega^{2} C_{a}\right) /\left(1+R^{2} \omega^{2} C_{a}^{2}\right)
\end{aligned}
$$

$\operatorname{tg} \delta=Z^{\prime} / Z^{\prime \prime}$, а $\varepsilon^{\prime}$ и $\varepsilon^{\prime \prime}$ даются выражениями (1), (2). Эта схема также не соответствует результатам эксперимента, поскольку частотная зависимость $\operatorname{tg} \delta(\omega)$ не имеет максимума.

\section{3. Одноконтурная схема}

Для одноконтурной схемы (рис. $3-3$ ) имеем усложненные выражения для $Z^{*}, \operatorname{tg} \delta, \varepsilon^{\prime \prime}$ и $\varepsilon^{\prime}$ по сравнению со схемами 1 и 2:

$$
Z^{*}=Z^{\prime}+i Z^{\prime \prime}
$$

где

и

$$
Z^{\prime}=R /\left[\left(\omega C_{a} R\right)^{2}+1\right]
$$

$$
\begin{aligned}
Z^{\prime \prime}= & {\left[\omega^{2} R^{2} C_{a}\left(C_{a}+C_{b}\right)+1\right] /\left[\omega C_{b}\left(\omega^{2} C_{a}^{2} R^{2}+1\right)\right], } \\
& \operatorname{tg} \delta=\left(R \omega C_{b}\right) /\left[1+R^{2} \omega^{2} C_{a}\left(C_{a}+C_{b}\right)\right] .
\end{aligned}
$$

Для функций $\varepsilon^{\prime \prime}(\omega)$ и $\varepsilon^{\prime}(\omega)$ могут быть получены аналитические выражения, не приведенные нами ввиду их громоздкости. Эти выражения могут быть тем не менее преобразованы к удобному для анализа виду путем исключения параметра $\omega$, что позволяет получить функциональную зависимость $\varepsilon^{\prime \prime}\left(\varepsilon^{\prime}\right)$. Эта зависимость имеет вид

$$
\begin{aligned}
& \varepsilon^{\prime \prime}=\left\{\varepsilon^{\prime} C_{g}\left(2 C_{a}+C_{b}\right) /\left[\left(C_{o}\left(C_{a}+C_{G}\right)\right)\right]\right. \\
& \left.-\left(\varepsilon^{\prime}\right)^{2}-C_{a} C_{b}^{2} /\left[C_{0}^{2}\left(C_{a}+C_{b}\right)\right]\right\}^{1 / 2}
\end{aligned}
$$

Зависимость (4) представляет собой полуокружность с двумя корнями для функции $\varepsilon^{\prime \prime}\left(\varepsilon^{\prime}\right): \quad \varepsilon^{\prime}=C_{b} / C_{0}$ (соответствует пределу при $\omega \rightarrow 0$ : правый корень функции $\varepsilon^{\prime \prime}\left(\varepsilon^{\prime}\right)$ на Коул-Коул диаграмме (рис. 1)) и $\varepsilon^{\prime}=\left(C_{a} C_{b}\right) /\left[\left(C_{a}+C_{b}\right) C_{0}\right] \quad$ (соответствует пределу при $\omega \rightarrow \infty$ : левый корень функции $\varepsilon^{\prime \prime}\left(\varepsilon^{\prime}\right)$ на КоулКоул диаграмме (рис. 1)). Здесь заметим, что ордината $h$ (радиус полуокружности) равна, как следует из выражения (4), полуразности корней функции $\varepsilon^{\prime \prime}\left(\varepsilon^{\prime}\right)$, т.е. $h=C_{b}^{2} /\left[2\left(C_{a}+C_{b}\right) C_{0}\right]$. Аналитическое выражение функции $\varepsilon^{\prime \prime}\left(\varepsilon^{\prime}\right)$ показывает также, что параметры Коул-Коул диаграммы зависят только от емкостей $C_{a}$ и $C_{b}$, но не зависят от $R$. В то же время функция $\operatorname{tg} \delta(f)$ имеет максимум на частоте $f_{0}=1 /\left[2 \pi R C_{a}\left(C_{b} / C_{a}+1\right)^{1 / 2}\right]$, положение которого зависит от $C_{a}, C_{b}$ и $R$.

Итак, извлекая из экспериментальной Коул-Коул диаграммы значения $\varepsilon_{1}^{\prime}(0)=9.43 \quad$ (при $\left.\omega \rightarrow 0\right)$ и $\varepsilon_{2}^{\prime}(0)=8.65$ (при $\omega \rightarrow \infty$ ), а из частотного положения максимума $\operatorname{tg} \delta(f)$ - частоту $f_{0}=10^{5} \mathrm{~Hz}$ (рис. 1), определяем по этим реперным точкам значения $C_{a}=170 \mathrm{pF}$, $C_{b}=13 \mathrm{pF}$ и $R=10 \mathrm{k} \Omega$.

В качестве иллюстрации к формулам (3) и (4) на рис. 1 для схемы 3 в виде графиков представлены сплошными линиями функция $\operatorname{tg} \delta(f)$ (3) и Коул-Коул диаграмма $\varepsilon^{\prime \prime}\left(\varepsilon^{\prime}\right)(4)$ для найденных значений параметров одноконтурной схемы. Экспериментальные точки хорошо соответствуют сплошным линиям графиков.

Заметим, что указанные значения параметров схемы были введены в пакет программ управляющего компьютера диэлектрического спектрометра. При этом были получены те же графики. Такой результат провоцирует соблазн добиться лучшего согласия расчетных и экспериментальных данных путем варьирования численных значений элементов схемы 3. Однако варьировать эти параметры не следует, хотя пакет программ спектрометра позволяет это делать. Запрет основан на том, что при описанном методе анализа данных степень соответствия расчетных кривых эксперименту говорит не о качестве подгонки, а о степени адекватности выбранной эквивалентной схемы реальным физическим свойствам образца. 


\section{4. Двухконтурная схема}

Для двухконтурной схемы 4 выражения для $Z^{*}, \operatorname{tg} \delta$, $\varepsilon^{\prime \prime}$ и $\varepsilon^{\prime}$ оказываются многократно более сложными, чем для схем $1-3$.

В целях упрощения процедуры записи выражений введем обозначения:

$$
\begin{array}{cc}
p=C_{b} R\left(C_{a}+C b\right)-C_{a} C_{b} R, & q=\omega^{2} R^{2} C_{b}^{2} C_{a}^{2}+C_{b}^{2}, \\
l=\omega^{2} C_{a} C_{b} R^{2}\left(C_{a}+C_{b}\right)+C_{b}, & s=\omega^{3} R^{2} C_{b}^{2} C_{a}^{2}+\omega C_{b}^{2}, \\
\alpha=C_{m} r\left(C_{m}+C_{n}\right)-C_{m} C_{n} r, & \beta=\omega^{2} r^{2} C_{n}^{2} C_{m}^{2}+C_{m}^{2}, \\
\sigma=\omega^{2} C_{m} C_{n} r^{2}\left(C_{m}+C_{n}\right)+C_{m}, & \rho=\omega^{3} r^{2} C_{m}^{2} C_{n}^{2}+\omega C_{m}^{2} .
\end{array}
$$

Тогда, полагая, что $A=P / q$ и $B=l / s$ представляют собой вещественную и мнимую части комплексного импеданса первого контура, а $M=\alpha / \beta$ и $N=\sigma / \rho-$ вещественную и мнимую части соответственно второго контура (рис. 3,4), получаем возможность записать выражение для полного импеданса в явном виде. В принятых обозначениях импеданс двухконтурной схемы имеет вид $Z^{*}=Z^{\prime}+i Z^{\prime \prime}$, где

$$
\begin{aligned}
& Z^{\prime}=\frac{[(A M-B N)(A+M)+(A N+B M)(B+N)]}{\left[(A+M)^{2}+(B+N)^{2}\right]}, \\
& Z^{\prime \prime}=\frac{[(A N+B M)(A+M)-(A M-B N)(B+N)]}{\left[(A+M)^{2}+(B+N)^{2}\right]} .
\end{aligned}
$$

Тангенс угла диэлектрических потерь равен $\operatorname{tg} \delta=Z^{\prime} / Z^{\prime \prime}$, а выражения для $\varepsilon^{\prime \prime}$ и $\varepsilon^{\prime}$ могут быть получены в параметрическом виде из формул (5), (6) и $(1),(2)$ (параметром является циклическая частота $\omega$ ). Из-за их громоздкости мы выражения для $\varepsilon^{\prime \prime}$ и $\varepsilon^{\prime}$ здесь не приводим.

Получение зависимости $\varepsilon^{\prime \prime}\left(\varepsilon^{\prime}\right)$ (Коул-Коул диаграммы) в явном аналитическом виде затруднительно ввиду необходимости отыскания общего вида корней полинома 8 степени. Однако имеется возможность построения Коул-Коул диаграммы на основе формул (1), (2), которые допускают параметрическое построение функции $\varepsilon^{\prime \prime}\left(\varepsilon^{\prime}\right)$ численными методами. На рис. 2 представлены результаты расчета функции $\operatorname{tg} \delta(f)$ и Коул-Коул диаграммы $\varepsilon^{\prime \prime}\left(\varepsilon^{\prime}\right)$ для эквивалентной схемы 4.

Результаты построения показывают, что частотная зависимость $\operatorname{tg} \delta(f)$ имеет два максимума, а Коул-Коул диаграмма состоит из двух полуокружностей. Эти кривые качественно совпадают с экспериментальными точками рис. 2. Схема 4 содержит 6 параметров: $R, C_{a}, C_{b}$ и $r, C_{m}, C_{n}$.

Переходя в выражениях для $Z^{\prime}(\omega)$ и $Z^{\prime \prime}(\omega)$ к пределу при $\omega \rightarrow 0$ (что соответствует правой абсциссе точки $\varepsilon^{\prime \prime}=0$ на Коул-Коул диаграмме) и вычисляя предельные значения $\varepsilon^{\prime}$ и $\varepsilon^{\prime \prime}$, получим из (5), (6) и (1), (2) выражение $\varepsilon^{\prime}=\left(C_{b}+C_{m}\right) / C_{0}$. Переходя к пределу при $\omega \rightarrow \infty$ (что соответствует левой абсциссе точки $\varepsilon^{\prime \prime}=0$ на Коул-Коул диаграмме) получим из (5), (6) и (1), (2) выражение

$$
\varepsilon^{\prime}=\left(C_{a} C_{b}\right) /\left[\left(C_{a}+C_{b}\right) C_{0}\right]+\left(C_{m} C_{n}\right) /\left[\left(C_{m}+C_{n}\right) C_{0}\right] .
$$

По аналогии со схемой 3 можно в первом приближении принять, что сумма „высот“ двух полуокружностей диаграммы равна полуразности корней функции $\varepsilon^{\prime \prime}\left(\varepsilon^{\prime}\right)$, T.e.

$$
\begin{aligned}
h_{1}+h_{2} & =0.5\left[\left(C_{b}+C_{m}\right) / C_{0}-\left(C_{a} C_{b}\right) /\left[\left(C_{a}+C_{b}\right) C_{0}\right]\right. \\
& -\left(C_{m} C_{n}\right) /\left[\left(C_{m}+C_{n}\right) C_{0}\right] .
\end{aligned}
$$

В рамках принятого приближения дополнительно предполагается, что слагаемые суммы $h_{1}+h_{2}$ являются независимыми друг от друга. При этом принимается, что „высота“ высокочастотной полуокружности равна $h_{1}=0.5 C_{b}^{2} /\left[\left(C_{a}+C_{b}\right) C_{0}\right]$, а „высота“ низкочастотной полуокружности равна $h_{2}=0.5 C_{m}^{2} /\left[\left(C_{n}+C_{m}\right) C_{0}\right]$. Такое предположение имеет смысл при условии, что центры полуокружностей „сильно разнесены“ по частоте $\omega$ и по абсциссе $\varepsilon^{\prime \prime}$.

Проверка справедливости предположения о независимости осуществлялась в настоящей работе следующим образом.

Во-первых, для схемы 4 в параметрическом виде по формулам (5), (6) и (1), (2) проводился расчет и построение графика зависимости $\mathcal{\varepsilon}^{\prime \prime}\left(\varepsilon^{\prime}\right)$.

Во-вторых, в данный расчет были введены 6 численных значений параметров элементов схемы 4, которые приведены в подписи к рис. 2. Оказалось, что „высоты“ $h_{1}=1.0$ и $h_{2}=0.75$ Коул-Коул диаграммы хорошо соответствуют численным значениям, полученным на основе выражений для $h_{1}=0.5 C_{b}^{2} /\left(C_{a}+C_{b}\right) C_{0}$ и $h_{2}=C_{m}^{2} /\left(C_{n}+C_{m}\right) C_{0}$. При этом их удвоенная сумма $\mathrm{c}$ хорошей точностью равна разности 3.5 корней функции $\varepsilon^{\prime \prime}\left(\varepsilon^{\prime}\right)$. Итак, благодаря использованию трех упомянутых реперных точек число варьируемых параметров удается сократить с шести до трех: $C_{b}, R$ и $r$.

Таким образом, извлекая из экспериментальной КоулКоул диаграммы значения $\varepsilon^{\prime}=10.1$ (при $\omega \rightarrow 0$ ), $h_{1}=1.0$ и $h_{2}=0.75$, а из экспериментальной зависимости $\operatorname{tg} \delta(f)$ частотные положения максимумов $f_{01}=10^{5} \mathrm{~Hz}$ и $f_{02}=4 \cdot 10^{3} \mathrm{~Hz}$, получаем (путем варьирования трех оставшихся параметров) численные значения элементов схемы, указанные в подписи к рис. 2. Эти значения соответствуют наилучшему согласию расчета с результатами измерений. На рис. 2 для схемы 4 в виде графиков представлены результаты расчета $\operatorname{tg} \delta(f)$ и Коул-Коул-диаграмма $\varepsilon^{\prime \prime}\left(\varepsilon^{\prime}\right)$ по формулам $(1),(2)$ для полученных значений параметров.

Заметим также, что указанные в подписи к рис. 3 значения элементов схемы 4 могут быть, как и для схемы 3, введены в пакет программ спектрометра, которые допускают их варьирование. Здесь в отличие от схемы 3 варьирование допустимо, но при условии постоянства численных значений параметров, соответствующих трем реперным точкам.

\section{5. Обсуждение результатов}

На основе сравнительного анализа данных диэлектрической спектроскопии и результатов макроскопическо- 
го моделирования электрического отклика пленок $\mathrm{VO}_{2}$ можно сделать следующие выводы.

1. Характеристики нелегированной пленки $\mathrm{VO}_{2}$ и процессы, происходящие в ней при приложении переменного внешнего напряжения, хорошо описываются одноконтурной схемой 3 (рис. 3). Для уравнений, описывающих эту схему, получено точное аналитическое решение уравнений, моделирующих электрический отклик. Путем сравнения результатов расчета с экспериментом по трем реперным точкам (которыми являются два корня функции $\varepsilon^{\prime \prime}\left(\varepsilon^{\prime}\right)$ и частота, соответствующая максимуму функции $\operatorname{tg} \delta(\omega))$, однозначно определены численные значения всех элементов модельной схемы.

2. Для моделирования диэлектрических спектров легированной германием пленки $\mathrm{VO}_{2}$ : Ge привлечена двухконтурная схема 4 (рис. 3). Математическое моделирование в этом случае усложнено по сравнению с одноконтурным вариантом, что вынуждает прибегнуть к параметрическому построению Коул-Коул диаграммы. Однако и в случае схемы 4 получена высокая степень соответствия результатов расчета с данными эксперимента, но уже при использовании процедуры подгоночного варьирования трех из шести параметров схемы.

3. Определена с высокой точностью электрическая емкость тонкой пленки диоксида ванадия. Для нелегированной пленки численное значение емкости оказалось равным $170 \mathrm{pF}$. Данный результат демонстрирует большие экспериментальные возможности метода диэлектрической спектроскопии, поскольку измерение стандартным способом электроемкости пленки толщиной $1400 \AA \AA$, синтезированной на подложке толщиной $0.5 \mathrm{~mm}$, практически невозможно ввиду многократного различия численных значений емкостей подложки и пленки, соединенных последовательно.

4. Легирование пленки $\mathrm{VO}_{2}$ германием приводит к уменьшению электрической емкости пленки и к разделению всей массы зерен пленки на две проникающие друг в друга совокупности: на совокупность слабо легированных зерен и на совокупность сильно легированных зерен. О соотношении суммарных площадей, занимаемых различными совокупностями зерен, можно судить по соотношению значений емкостей $C_{b}=7 \mathrm{pF}$ и $C_{m}=5 \mathrm{pF}$, легко определяемых методом диэлектрической спектроскопии.

5. Для обеих совокупностей электрические сопротивления зерен повышаются при легировании, однако для сильно легированных зерен повышение в несколько раз больше, чем для слаболегированных $(r=3 \mathrm{M} \Omega$ и $R=60 \mathrm{k} \Omega$ соответственно (для нелегированных зерен $R=10 \mathrm{k} \Omega)$ ). Для обеих совокупностей суммарные значения емкостей при легировании уменьшаются, причем как для сильно легированных, так и для слаболегированных зерен $\left(C_{n}=12 \mathrm{pF}\right.$ и $C_{a}=15 \mathrm{pF}$ соответственно (емкость совокупности нелегированных зерен $\left.\left.C_{a}=170 \mathrm{pF}\right)\right)$.

Таким образом, спектроскопические методы позволяют выделить из общей массы зерен пленки $\mathrm{VO}_{2}$ две независимые совокупности, каждой из которой присущи свои электрические характеристики. Проделанный анализ показал, что легирование пленок $\mathrm{VO}_{2}$ германием приводит к изменению свойств всех зерен пленки, однако только для части нанокристаллитов пленки сопротивление легированных зерен повышается значительно. Остальная часть нанокристаллитов пленки свои характеристики меняет слабо. Причиной повышения сопротивления легированных зерен является уменьшение в них концентрации свободных электронов. Дело в том, что ионы $\mathrm{Ge}$ в решетке $\mathrm{VO}_{2}$ обладают „акцепторными“ свойствами, несмотря на то, что они являются изоэлектронными по отношению к ионам $\mathrm{V}^{4+}$, которые замещают при легировании. Анализ показывает, что при своем встраивании в кристаллическую решетку $\mathrm{VO}_{2}$ ион $\mathrm{Ge}$ формирует такую конфигурацию своих гибридных электронных орбиталей, что часть из них остается свободной, не участвуя в создании химических связей с решеткой. Захватывая на свободные орбитали электроны из зоны проводимости, ион $\mathrm{Ge}$ снижает в ней концентрацию свободных носителей и увеличивает тем самым электрическое сопротивление кристаллита. В дополнение к этому в таком сильнокоррелированном материале, как диоксид ванадия, данный эффект дополнительно усилен благодаря корреляционному росту запрещенной зоны [4].

В заключение отметим, что диэлектрическая спектроскопия является высокочувствительным экспрессметодом анализа электрических свойств тонких кристаллических пленок. В частности, для легированных пленок диэлектрическая спектроскопия является эффективным методом определения электрофизических свойств совокупностей нанокристаллитов, сильно различающихся степенью легирования.

\section{Конфликт интересов}

Авторы заявляют, что у них нет конфликта интересов.

\section{Список литературы}

[1] Castro R.A., Ignatiev A.I., Nikonorov N.V., Sidorov A.I., Stolyarchuk M.V. // J. Non-Crystalline Solids. 2017. Vol. 461. P. 72-79.

[2] Kremer F., Schonhals A. Broadband Dielectric Spectroscopy. Berlin: Springer, 2003.

[3] Ильинский А.В., Кастро Р.А., Никулин Е.И., Шадрин Е.Б. // ЖТФ. 2018. Т. 88. Вып. 6. С. 877-882.

[4] Ильинский А.В., Пашкевич М.Э., Шадрин Е.Б. // НТВ СПбГПУ. ФМН. 2017. Т. 10. Вып. 3. С. 9-17.

[5] Ильинский А.В., Кастро Р.А., Кононов А.А., Пашкевич М.Э., Попова И.О., Шадрин Е.Б. // Письма в ЖТФ. 2019. Т. 45. Вып. 11. С. 44-46.

[6] Калашников С.Г. Электричество. 6-е изд. Изд-во: ФМЛ, 2004. 